\title{
Materias optativas como reforzadores de la identidad de normalistas
}

\author{
Felisa Escoto Murillo \\ Melania Elizabeth Pérez Rodríguez \\ Silvia Lizette Ramos de Robles \\ Benemérita y Centenaria Escuela Normal de Jalisco
}

\section{Resumen}

1 1 presente estudio se desarrolló en $\checkmark$ la Benemérita y Centenaria Escuela Normal de Jalisco, el propósito general fue identificar y describir desde la perspectiva émica, la construcción de la identidad del estudiante normalista. El análisis se abordó desde el papel que juegan los talleres (actividades extracurriculares) como reforzadores de la identidad. Para documentarlo se utilizaron los planteamientos de la perspectiva de investigación cualitativa a través del estudio de caso, el cual aporta las bases idóneas para seleccionar al sujeto de estudio dentro de un escenario real. La elección de casos fue de tipo no probabilístico ya que los participantes son voluntarios. Los instrumentos que apoyan en la recolección de los datos son: la técnica de la asociación libre (redes semánticas) y la entrevista semiestructurada. Para el caso de referentes teóricos se retoman conceptos clave como: identidad, representaciones sociales y comunidad de práctica. Los resultados permiten identificar que los talleres: a) constituyen espacios de convivencia en los cuales los normalistas fortalecen sus lazos identitarios y donde se construyen comunidades de práctica con bases sólidas que provienen de sus intereses personales, académicos y de recreación artístico-cultural; b) aportan más elementos para la identidad profesional que los espacios curriculares obligatorios.

Palabras clave: formación del profesorado, identidad, talleres. 
REVISTA DE INVESTIGACIÓN EDUCATIVA DE LA REDIECH N. 7 ISSN: 2007-4336

\section{Introducción}

La palabra extracurricular es un constructo mediante el prefijo extra que significa "fuera de", o "en el exterior"; dentro del contexto de la Benemérita y Centenaria Escuela Normal de Jalisco (ByCENJ), la definiremos como aquella materia que se cursa fuera de un itinerario curricular, es decir, toda aquella asignatura que sea complementaria a las establecidas en los planes y programas de estudio. Estas materias ofrecen la oportunidad al alumno de responsabilizarse de su proceso de aprendizaje porque eligen cómo ir estructurando su perfil de egreso.

Con respecto a la educación complementaria de los estudiantes de la Licenciatura en educación primaria, el Plan y Programa de la SEP (1997:47) en su apartado número nueve menciona "en la formación integral del maestro es indispensable el desarrollo de sus capacidades de expresión y apreciación artísticas, así como la adquisición de hábitos y aficiones relativas a la educación física y las prácticas deportivas", por tanto, las materias optativas además de complementar los conocimientos, las destrezas y las competencias, les permitirá fortalecer características que son propias de la identidad que como normalistas construyen durante su formación.

Elisondo, Donolo y Rinaudo (2009) mencionan que las actividades extracurriculares deben ser diversas, variadas y opcionales, entre las que los alumnos puedan escoger según sus motivaciones e intereses, la oferta de estas materias además de satisfacer las necesidades del estudiante debe ser el complemento de la malla curricular.
En una búsqueda general sobre investigaciones relacionadas con este tema, se encontró que existen diversos estudios que abordan las materias optativas desde varios enfoques con la intención de clarificar su función y sentido, y la manera en que ellas caracterizan al currículum, de cómo se diseñan y cuáles son sus características desde la práctica misma.

La importancia de estas actividades es su contribución para lograr una formación más amplia en los alumnos; Araujo (2010:1) menciona que "este tipo de actividades promueven en el alumno un sentimiento de pertenencia al centro y al grupo, ayudan a conseguir que adquieran una mayor autonomía y responsabilidad en la organización de su tiempo libre", de esta manera se favorecen y refuerzan sus habilidades sociales y comunicativas además de mejorar las relaciones entre estudiantes.

Barboza, Martínez y Mendizabal (2012: 6) las catalogan como estrategias de reforzamiento a la formación y al aprendizaje y no todas son obligatorias, designan con el nombre de

...cocurriculares a aquellas cuya realización está señalada en el calendario y se consideran complementarias a los contenidos curriculares para el logro de los aprendizajes esperados, y extracurriculares a las que no guardan una relación directa con los contenidos y su realización y que no se mencionan en el calendario pero que coadyuvan de manera importante a la formación de los alumnos.

En el contexto universitario las materias optativas toman un especial interés, como lo muestra la investigación realizada por 
Méndez (s/f) dado que aportan características a ciertas materias y su diseño se pretende fortalecer la malla curricular.

En este contexto, la identidad estudiantil es un tema de relevancia para las instituciones de educación superior. El comprender el grado de identidad de los estudiantes sirve para generar estrategias que produzcan una mayor identificación del alumnado hacia su institución, que impulse el crecimiento institucional hacia dentro y fuera de la misma, para que las prácticas sociales que establecen y el sentido de pertenencia dentro de su institución formadora sean los dos elementos primordialmente determinantes.

Por tanto, la presente investigación aborda como objeto de estudio los talleres, con el único fin de establecerlas como vínculo para fortalecer la identidad normalista de los alumnos de la BYCENJ.

\section{Propósito}

Con el presente estudio se pretende identificar los elementos de las materias optativas que refuerzan la construcción de la identidad del alumno normalista, desde la propia opinión de los estudiantes.

\section{Marco Teórico}

Plantear el tema de identidad supone transitar por su multidimensionalidad y lo complejo del término que comprende lo cultural, social, psicológico, sexual, personal, entre otros; con lo que nos encontramos colocados frente a un conjunto de situaciones que inciden directamente en la forma en que cada uno de nosotros nos concebimos en los diversos ámbitos de actuación. Por tanto, debemos asumir un concepto de identidad dinámica y progresiva de acuerdo a las interacciones en las que estamos inmersos como sujetos sociales.

La identidad también es definida por Erickson como "un sentimiento de mismidad y continuidad que experimenta un individuo en cuanto tal" (citado por Mercado y Maldonado, 2010); es decir, el individuo tiene que reflexionar sobre la pregunta ¿Quién soy? y como resultado, se reconoce a sí mismo y da cuenta de sus características propias que lo hacen único.

Pero la identidad va más allá de la individualidad, Dubar (1991) afirma que la identidad no es otra cosa que el resultado estable y provisorio, individual y colectivo, subjetivo y objetivo, biográfico y estructural de los diversos procesos de socialización que conjuntamente construyen los individuos y definen las instituciones. De este modo, la identidad es lo subjetivo, pero también lo social, son las pertenencias y exclusiones, las afinidades y diferenciaciones, las cercanías y distanciamientos.

La identidad está vinculada a la concepción de sociedad y a la percepción que se tiene de la propia posición dentro de ésta; también las expectativas, los valores y las normas forman parte del mismo proceso unitario de conformación de la identidad.

La identidad no tiene un concepto específico, si se considera que ha sido abordado desde el punto de vista de distintos autores y cada disciplina le da su propio enfoque, y porque los individuos construimos nuestra identidad a medida que vivimos, por lo tanto, está sujeta a redefiniciones. Para esta investigación fue necesario apoyarse 
de estos elementos para crear un concepto propio:

Identidad es la representación misma del sujeto que identifica sus características, en relación con los otros, haciéndolo diferente y necesario para pertenecer a un grupo que comparte rasgos y que a su vez lo definen como integrante de éste.

Con este sentido de pertenecer o de ser parte, es como nace la identificación colectiva de forma institucionalizada, Wenger (2001:99) lo define como: comunidad de práctica: "conjunto de individuos que comparten una cultura y prácticas definidas, cuya interacción es dimensional, es decir, cada miembro tiene diferente forma de participación pero una misma empresa”. Así mismo, refiere a tres dimensiones de la práctica como propiedades de una comunidad: compromiso mutuo, empresa conjunta y repertorio compartido, donde se advierte una semejanza entre las dimensiones de Wenger con las diversas acciones del plan de desarrollo institucional, como se describen en la tabla 1:.

Dimensiones de la comunidad de práctica (Wenger 2001) Plan de Desarrollo Institucional 1997 (PDI)

\begin{tabular}{|l|}
\hline Dimensiones de Wenger \\
Compromiso mutuo: La afiliación de \\
los participantes es definida por su \\
compromiso, dando así, la cohesión \\
necesaria para funcionar aunque \\
existan diferencias y similitudes entre \\
sus miembros, logrando un lugar \\
único para cada uno y adquirir una \\
identidad propia con base en estas \\
interacciones.
\end{tabular}

Empresa Conjunta: Las comunidades de práctica tienen como objetivo producir su propia práctica manteniéndola unida como comunidad dando origen a relaciones de responsabilidad mutua entre los participantes.

Acciones de la comunidad normalista

Misión institucional: Dentro de la comunidad normalista de la ByCENJ, prevalece un compromiso mutuo entre todos los integrantes que la conforman desde personal de apoyo, administrativos, maestros, alumnos y directivos, desempeñan sus funciones con la empresa de "Ofrecer un servicio educativo de excelencia académica orientado a la formación de Licenciados en Educación Primaria, que desarrolla a plenitud las competencias profesionales de los normalistas en el cumplimiento de su perfil de egreso; la sensibilidad hacia las labores de enseñanza, la creatividad y la flexibilidad hacia el entorno como insumo para el aprendizaje, la tendencia hacia la innovación pedagógica y el desarrollo integral del ser humano; y la actualización docente fincada en la observación de los valores y principios del laicismo educativo".

Visión Institucional: "Somos una Institución educativa con más de cien años de experiencia en la formación inicial de profesores de educación primaria, que aspira a cumplir de manera cotidiana su compromiso social de actualización permanente para que cada uno de sus egresados sepa responder exitosamente a las necesidades de aprendizaje de los niños y las niñas de la escuela primaria. Fundamentamos nuestro quehacer docente en un clima de trabajo colaborativo basado en el estudio sistemático y en el mejoramiento continuo de las competencias profesionales establecidas en el plan de estudios 1997 de la Licenciatura en Educación Primaria".

Repertorio Compartido: La actuación conjunta encaminada a la consecución de una empresa crea recursos para negociar significados, estos elementos que incluye rutinas, palabras, instrumentos, maneras de hacer, relatos, gestos, símbolos, géneros, acciones o conceptos que la comunidad ha producido o adoptado en el curso de su existencia y que han pasado a formar parte de su práctica.
La jornada de práctica.

Actos cívicos.

Logotipo de la institución en la vestimenta.

Áreas emblemáticas de la institución: mural, teatro griego, aula magna, materias optativas, entre otros.

Tabla 1. Relación entre las dimensiones desde la teoría de Wenger y las acciones de la comunidad normalista 
Con base en la relación que existe entre el concepto de las dimensiones del autor y las acciones del Plan de Desarrollo Institucional de la ByCENJ, tiene sentido llamar a la comunidad de práctica como comunidad normalista específicamente la escolar.

Pertenecer a la comunidad normalista implica conocer los distintas factores que son propios de la misma como: experiencias escolares pasadas, relaciones humanas, afinidades, percepción de su institución, ingreso, egreso, reglas, educación, este último es en el que nos enfocaremos para abordar las materias optativas.

Por lo que, atendiendo al Plan y Programa de la Licenciatura en Educación Primaria (1997) en su apartado número nueve, la ByCENJ ofrece las materias optativas o talleres extracurriculares. También sugiere que para promover las capacidades antes mencionadas "se disponga de tiempos programados que ofrezcan elementos comunes de formación y actividad a los estudiantes, así como de tiempos adicionales que puedan ocuparse de manera flexible y sin programación rígida" (SEP, 1997:47) Es aquí donde la institución presenta la gama de posibilidades extracurriculares, la mayoría en horarios contra turno para los alumnos normalistas.

Estas materias han ido adquiriendo un lugar importante, ya que han repercutido en la imagen, el prestigio y la calidad dentro y fuera de la ByCENJ, a corto y largo plazo, propiciando en la comunidad normalista escolar una mayor identificación hacia su institución.

El vínculo que se propicia a partir de la identificación, es un factor de importancia, es la relación con un objeto, fruto del cual aparece una conducta más o menos fija con ese objeto, que forma un patrón o pauta de conducta que tiende a repetirse automáticamente, tanto en la relación interna como en la relación externa con el objeto (Riviere, 2012).

Mercado y Hernández (s/f) citan a Tajfel, el cual desarrolla una teoría de la identidad social, concibiéndola como el vínculo psicológico que permite la unión de la persona con su grupo, "la pertenencia al grupo es el ingrediente esencial de la identidad social, porque al mismo tiempo que se siente parte de un grupo, el individuo se diferencia de los miembros de otros grupos a los que pertenece", por lo que la identificación del estudiante se da por la adscripción al grupo, ya que cuando comprueba que es diferente a otros se reafirma la pertenencia al mismo.

Prueba clara son las materias optativas consideradas emblemáticas para la institución formadora de docentes como: las bastoneras, el orfeón, la banda de guerra, la escolta, la estudiantina, animación, entre otras, que le han dado a la institución proyección hacia el exterior, tienen un reconocimiento de las autoridades gubernamentales de nuestra ciudad y en la que los estudiantes adscritos a ellas, adoptan ciertas características que la comunidad normalista les reconoce.

Estas dinámicas sociales que se desarrollan a partir de pertenecer a un grupo de taller optativo, sin dejar de lado su devenir escolar cotidiano, genera y reafirma la identidad social en cada uno de los estudiantes y lo define en su relación con otros, 
compartiendo un macro contexto escolar.

A través de la interacción de estos factores y las manifestaciones más o menos estables de éstos dentro de un contexto institucional y social, se construye la identidad estudiantil (Cabral y Villanueva, 2006).

\section{Metodología}

La investigación fue realizada en la Benemérita y Centenaria Escuela Normal de Jalisco, en la que participaron de forma voluntaria 16 estudiantes de cuarto semestre de la generación 2011-2015.

La muestra se inclinó por los estudiantes del cuarto semestre ya que están plenamente inmersos en las dinámicas tanto académicas como sociales de su institución formadora, se identifican con sus iguales y con el grupo al que pertenecen, comparten experiencias personales y propias de un estudiante normalista, así mismo discriminan estas experiencias desde su llegada a la institución, el lugar que tienen en el presente y el que desempeñarán en semestres posteriores.

Cabe resaltar que durante el tercer semestre, los estudiantes de la misma generación, participaron en la aplicación de la prueba piloto de los instrumentos, con la finalidad de comprobar la confiabilidad y validez de los mismos.

Para la recolección de datos se utilizaron: la técnica de la asociación libre como un primer acercamiento a lo más representativo para los estudiantes con respecto a la identidad normalista. Se convocó a los sujetos de estudio, previa invitación, para la aplicación de la técnica de asociación libre.
- Se solicitó de manera voluntaria un estudiante de cada grupo de cuarto semestre, para acudir a la aplicación del primer instrumento para la recolección de datos, siendo un total de 16 estudiantes de ambos turnos.

- Se dieron indicaciones de cómo realizar adecuadamente la técnica y se proporcionó un ejemplo.

Se pronunció la frase generadora "identidad normalista".

- El estudiante expresó de manera escrita en cinco palabras, lo primero y más significativo que le representara la frase generadora.

- Los participantes jerarquizaron las palabras anteriormente escritas, es decir, les dieron un valor numérico a estas palabras.

Se dio por concluida la técnica de asociación libre.

\section{Redes semánticas naturales}

Las redes semánticas naturales contribuyeron a analizar el conjunto de palabras resultantes de la técnica de asociación libre y, además, fueron el complemento del análisis semántico de dichas palabras para la construcción de la misma, y expresar de manera esquemática esta concepción.

En nuestro país, la técnica de redes semánticas naturales es ampliamente usada en el campo educativo, dado que los significados del objeto de estudio son una abstracción de un concepto con base en una conducta, la red semántica natural es la técnica adecuada pues funge como media- 
dora entre el objeto de estudio y la conducta observable de los estudiantes.

En este sentido, se buscó que expresaran el significado de la concepción "identidad normalista" a través de red de asociaciones con las palabras obtenidas, cada uno de los nodos que en esta red se expresan guardan relación y además constituyen propiedades del propio objeto de estudio.

\section{Entrevista semiestructurada}

En el transcurso de la investigación se planeó la utilización de la técnica de entrevista semiestructurada como un recurso complementario en la recolección de información que contribuya al cumplimiento del objetivo, es decir, a describir la construcción de la identidad normalista de los alumnos del cuarto semestre de la Benemérita y Centenaria Escuela Normal de Jalisco.

El proceso de aplicación se llevó a cabo en tres momentos, equivalentes a las participaciones de cada uno de los investigadores, donde al mismo tiempo intervenía otro como observador para tomar nota de los hechos no verbales que surgían durante las mismas, con la finalidad de comprobar la intención del argumento mediante el lenguaje no verbal.

A partir de la estructura de la red semántica natural y apoyados en los nodos del esquema, se diseñaron las categorías que dieron paso al guión de la entrevista semiestructurada que se aplicó en la última fase, para clarificar y ampliar el concepto de identidad, durante la recolección de información.

\section{Análisis de los datos cualitativos}

Después de recoger los datos, el análisis es la fase en la que se procesa la información mediante actividades que se interrelacionan, es decir, cuando concluye una etapa se prosigue con la siguiente, pudiendo regresar a la anterior. El análisis de los datos cualitativos se basa en tres fases: $r e$ ducción de datos, disposición y transformación de datos y obtención y verificación de conclusiones.

\section{Resultados}

\section{Asociación libre}

Se consideró la importancia de la aparición de cada una de las palabras, multiplicándola por la frecuencia de las respuestas. Por lo tanto, el tratamiento de los resultados obtenidos se ven reflejados en el gráficol.

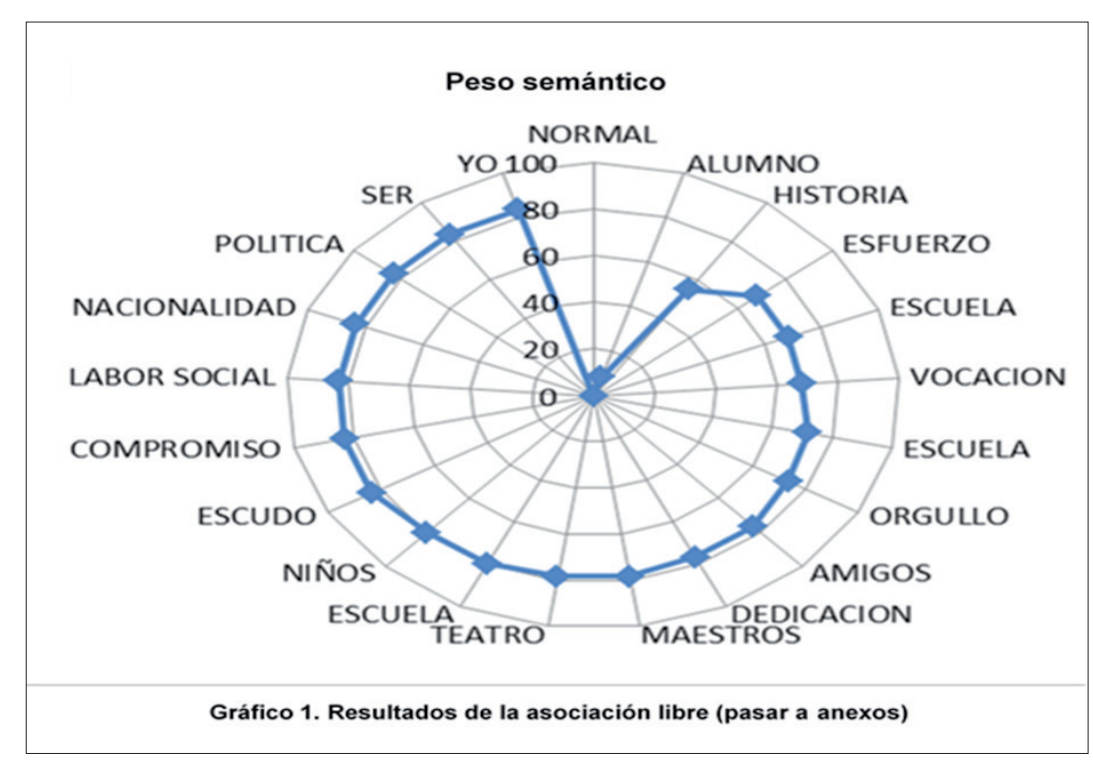


Se muestra a simple vista la manera en que las palabras definidoras se acercan al concepto "identidad normalista" (frase generadora) de los estudiantes. Cabe hacer mención que la gráfica se generó mediante una hoja de cálculo de Excel.

\section{Redes semánticas naturales}

A continuación los resultados se agruparon en categorías semánticas y a partir de ahí se dio origen a los nodos, entendidos como conceptos centrales. A través de la red de asociaciones con las palabras que se obtuvieron, cada uno de los nodos que en esta red se expresan, guardan relación y constituyen propiedades del propio objeto de estudio.
Se puede observar que el gráfico 2 contiene siete nodos, cada uno de ellos con diferente cantidad de palabras y a su vez, con un peso semántico distinto entre sí, que guarda relación con la palabra generadora, esto contribuye a expresar de manera visual cómo los individuos comprenden el concepto de "identidad normalista" y qué características le asignan.

\section{Entrevista semiestructurada}

Partiendo de la red semántica natural fue evidente notar los nodos que representan los conceptos más significativos para la muestra seleccionada, por lo que fueron considerados para siete categorías: relacional, signos o significados, contextual, institucional, profesional, valoral, individual.

\section{Red Semántica Natural}

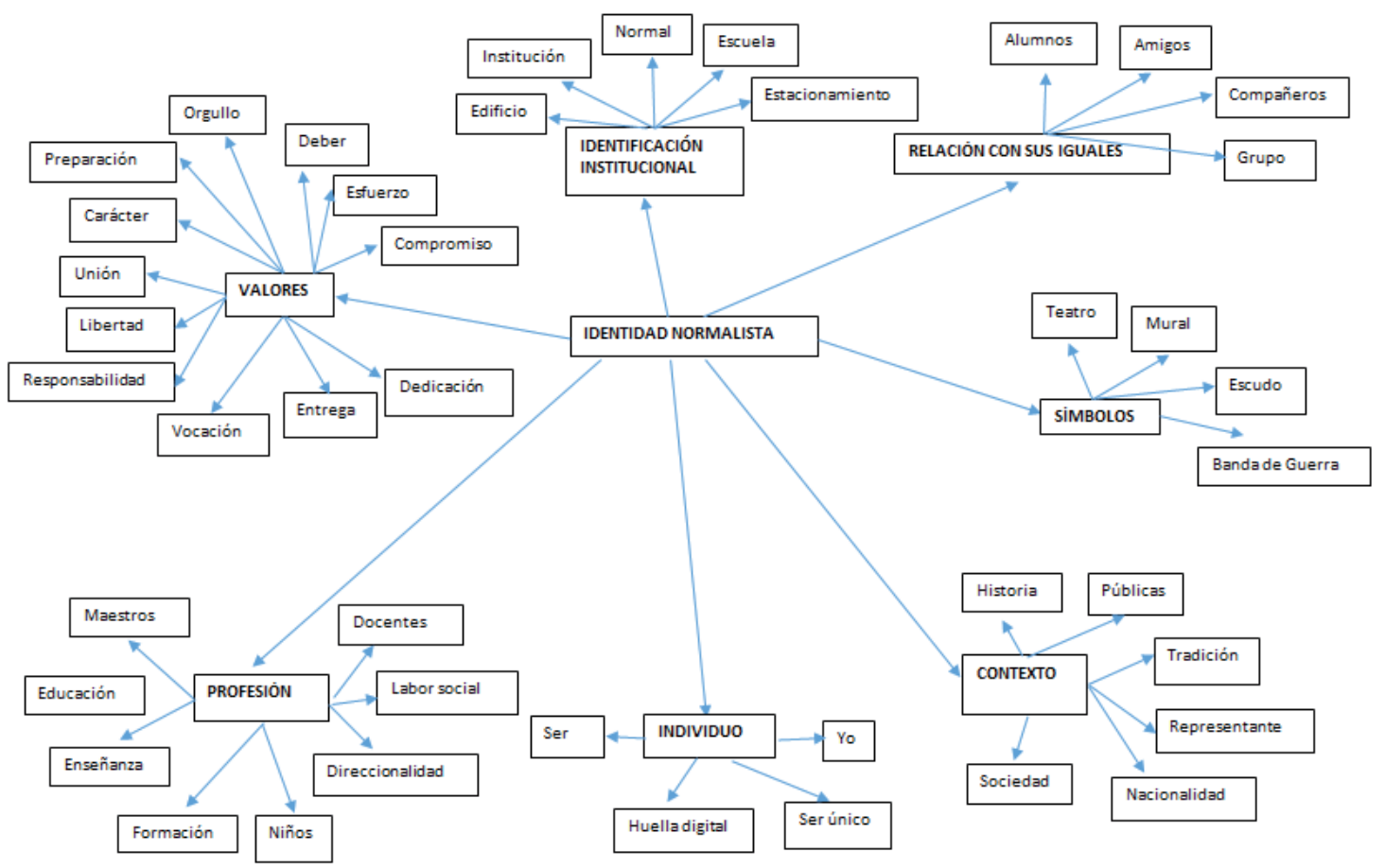

Gráfico 2. Red semántica natural de los resultados de la asociación libre 
Estas categorías direccionaron el guión para la entrevista semiestructurada. Posteriormente, la información que se recolectó fue objeto de procesamiento, se transcribieron 16 entrevistas, en el procesador de textos Microsoft Word para su tratamiento. Fue necesario recurrir a un software específico para datos cualitativos llamado MaxQDA 10. Este programa permitió establecer el agrupamiento de la información de las declaraciones de los entrevistados mediante códigos a los cuales se les asigna un color diferente a cada uno, y también permitió crear subcategorías.

Como producto de este análisis se presentan los siguientes resultados:

Para los estudiantes normalistas, las materias extracurriculares, llamadas también talleres optativos, han sido, además de motivadoras, el punto de encuentro de los estudiantes entre los diversos semestres. Dubar (1991) menciona que la identidad no es otra cosa que el resultado estructural de los diversos procesos de socialización que conjuntamente construyen los individuos.

Por otra parte han contribuido a mejorar su desempeño en las actividades académicas, brindan una identificación hacia su institución formadora y le dan prestigio al exterior de ella. Mercado y Hernández (s/f) puntualizan que la pertenencia al grupo es el ingrediente esencial de la identidad social, porque al mismo tiempo que se siente parte de un grupo, el individuo se diferencia de los miembros de otros grupos a los que pertenece.

Mostraron que el alumno ingresa a la institución con la firme intención de ocu- parse de sus estudios, de tener las mejores calificaciones, para aspirar en un futuro a un empleo que le dará una estabilidad económica y podrá obtener su independencia familiar, por lo que el estudiante deja de lado las relaciones sociales, ya que considera que no le benefician.

Por otra parte, puede advertirse que a través de la interacción, de las manifestaciones más o menos estables de los estudiantes dentro de un contexto institucional y social, las materias optativas son el vínculo entre los normalistas de diversos semestres y fortalecen la identidad estudiantil, los estudiantes refieren que éstas no son las más deseables para su elección, además que la oferta de estos talleres no cumple con los intereses del alumnado.

"Durante la clase del orfeón, de estudiantina, de bastoneras, de inglés coincidimos con compañeros de otros semestres y es donde socializamos además de aprender" (alumna de cuarto semestre).

Por último, y dado que cada vez las materias optativas son menos, surge la preocupación de que los únicos puntos de encuentro de los alumnos se vean nulificados y que se favorezca una sociedad de alumnos ajenos a la socialización, a la convivencia, a compartir y aportar, a ser parte de y a sentirse identificados con su institución formadora, y que la identidad normalista adopte características similares o iguales a las de las universidades, dejando en el olvido las que distinguen al normalista, al maestro humano, cálido y preocupado por atender las necesidades tanto académicas como socioculturales de sus alumnos. 
Por tanto, las cuestiones son ¿será benéfico o contradictorio eliminar las materias optativas? si éstas son el punto de encuentro y de socialización ¿qué tipo de estudiantes formará la Normal de Jalisco?, que sean trabajadores de la educación o docentes cuya formación integral aporta a sus alumnos no únicamente el camino para adquirir los conocimientos deseados, sino el que además se preocupa por la materia prima de su trabajo: niños con distintas necesidades, que van desde las especiales hasta los que no reciben la atención en casa.

\section{Referencias}

Araújo, N. (2010). Actividades complementarias y extraescolares. Gogomar. En:http://www.google.com. $\mathrm{mx} / \mathrm{url}$ ? $\mathrm{sa}=\mathrm{t} \& \mathrm{rct}=\mathrm{j} \& \mathrm{q}=\& \mathrm{esrc}=\mathrm{s} \&$ frm $=1$ \&source $=\mathrm{W}$ eb\&cd=1\&ved=0CBwQFjAA\&url=http $\% 3 \mathrm{~A} \% 2 \mathrm{~F} \%$ 2Fwww.edu.xunta.es\%2Fcentros\%2Fceipchanopin heiro\%2Fsystem\%2Ffiles\%2Fact.\%2520complemen tarias\%25202010.pdf\&ei=u91_VNTkC42SyQSdgIK wBQ\&usg=AFQjCNFKIDuo6jHm_zMo6nVYLEDf0vAIfw
Barbosa, J. Martínez, R. Mendizábal, R. (2012). Estrategias de reforzamiento a la formación y el aprendizaje. Telesecundaria. Dirección general de materiales educativos. Subsecretaría de educación básica. Secretaría de educación Pública. México.

Cabral, M., Villanueva, E., Quevedo, M. y Estrada, G. (2006). Escala de Identidad Estudiantil Universitaria.

Dubar, C. (2001). El trabajo y las identidades profesionales y personales. Revista Latinoamericana de Estudios del Trabajo. Año 7, No 13. pp. 5-16.

Elisondo, Donolo y Rinaudo (2009). Innovación educativa en la enseñanza formal. Edit. Universidad de Murcia.

Méndez, A. (s/f). ¿Cuál es el sentido de las materias optativas dentro del currículum de la Escuela de Psicología. Revista Vida Universitaria.

Mercado, A. (2010). El proceso de construcción de la identidad colectiva. En Convergencia. Revista de ciencias sociales. Núm. 53, mayo-agosto. pp. 229-251. Universidad Autónoma del Estado de México.

Riviere, E. (2012). Teoría del vínculo. Revista electrónica de Psicología Social. Núm. 23, junio. Universidad Nacional de Córdoba.

SEP (1997). Planes y programas de estudio para la $\mathrm{Li}$ cenciatura en Educación Primaria. Edit. SEP. México.

Wenger, E. (2001). Comunidades de Práctica, aprendizaje, significado e identidad. Ed. Paidós. México. 